\title{
Propuesta de enseñanza del Tenis de Mesa en Educación Primaria y Secundaria Proposal to the table tennis teaching in primary and secondary school
}

\author{
Manuel Tomás Abad Robles \\ C.E.I.P. Dunas de Doñana, Matalascañas - Huelva (España)
}

\begin{abstract}
Resumen: El Tenis de Mesa es un deporte que cada vez se practica más en todo el mundo, tanto en el campo federativo y competitivo como en el educativo y recreativo. La utilización, cada vez más frecuente, del Tenis de Mesa como contenido en las clases de Educación Física en centros de enseñanza Primaria y Secundaria, hace que cobre especial importancia su adecuada enseñanza, puesto que a través de su práctica se puede formar a la persona en los distintos ámbitos: motor, afectivo, cognitivo y social. Enseñanza que, en nuestra opinión, debe partir de las características del deporte y de sus potencialidades educativas, aunque como es bien sabido, el deporte puede ser, o no, educativo, dependiendo de la utilización que hagamos del mismo. Por lo que, y siguiendo a Tabernero, Márquez, y Llanos (2002), la figura clave de la iniciación deportiva va a ser el técnico/profesor. Por este motivo, abogamos por una práctica educativa del Tenis de Mesa.
\end{abstract}

Palabras clave: deporte, iniciación deportiva, educación, Tenis de Mesa.

Abstract: The table tennis is a sport which is practised increasingly in the world, so much in the federative and competitive fields as educative and recreative ones. The frequent use of the table tennis as content in the physical education lessons makes that its suitable teaching receives special importance, because with its practice we can educate people in different fields: motor, affective, cognitive and social. We think that this teaching must begin from characteristics of the sport and its educational possibilities, even though, as it is known, sport can be, or not, educative, depending on the use that we made of it. Following to Tabernero, Márquez, y Llanos (2002), the main person will be the coach/teacher in the sports initiation. For this reason we defend an educational practice of the table tennis.

Key words: sport, sports initiation, education, table tennis.

\section{Introducción}

En la actualidad, y siguiendo a Robles (2005), Salina y Viciana (2006), la enseñanza del deporte suele ser el contenido más utilizado dentro de las clases de Educación Física. En general se acepta que el deporte es un contenido que contribuye a la formación de los alumnos y alumnas, tanto en Primaria como en Secundaria: ayuda a desarrollar valores positivos y actitudes adecuadas, fomenta el trabajo de las cualidades afectivo-emocionales, físicas, cognitivas y sociales, así como colabora en la adquisición de hábitos higiénicos y saludables relacionados con la práctica deportiva en general. De esta forma, siguiendo a Giménez y Rodríguez (2006), pensamos que el Tenis de Mesa, como contenido educativo de la iniciación deportiva, puede ser utilizado en nuestras clases de Educación Física para favorecer la formación integral de nuestros alumnos y alumnas. Esto cobra importancia, al ser el Tenis de Mesa, según González y Aznar(2007), un contenido muy utilizado y conocido por nuestros pupilos.

A continuación exponemos los medios técnico-tácticos fundamentales que deben ser enseñados en la etapa de iniciación al Tenis de Mesa; no pretendemos describirlos exhaustivamente, puesto que ése no es el objetivo de este trabajo, sino nombrar aquéllos que son imprescindibles en la fase de formación de los jugadores. En este sentido, Angelescu (1988), al referirse a los medios técnicos que han desarrollarse en la enseñanza del Tenis de Mesa, realiza la siguiente propuesta, a la cual hemos añadidos otros aspectos a considerar:

1. Empuñadura de la pala

2. Familiarización con la pala y la pelota

3. Posición de base; saque

4. Devolución de los saques

5. Juego de piernas

6. Peloteo sin efecto de derecha y de revés

7. Peloteo cortado de derecha y de revés

8. Peloteo en semivolea de derecha y de revés

9. Corte de derecha y de revés

10. Ataque de derecha y ataque de revés

11. Golpe de contraataque de derecha y de revés

Fecha recepción: 25-06-07 - Fecha envío revisores: 29-06-07 - Fecha de aceptación: 06-11-07 Correspondencia: Manuel Tomás Abad Robles

$\mathrm{C} /$ Jilguero, $30 \mathrm{~B}$

21110 Aljaraque - Huelva (España)

E-mail: mtarobles@hotmail.com
12. Top spin de derecha y de revés

13. Devolución del top spin (blocaje de derecha y de revés)

14. Dejada

Por otro lado, el Manual de laF.E.T.M., Bermejo(1991), recomienda enseñar, tanto los golpes de ataque, como los de defensa; haciendo constar que hoy en día el juego de ataque es el que prevalece, pero que es conveniente que los jugadores tengan un conocimiento general, tanto de los golpes de ataque, como de los de defensa; consideración con la que estamos totalmente de acuerdo.

Normalmente, cuando consultamos un manual específico de Tenis deMesa, comprobamos que, en lo referente a la metodología propiamente dicha, solo se dan algunas pinceladas (en el mejor de los casos), haciéndose más hincapié en aspectos como descripción detallada de los gestos técnicos a seguir, etapas o fases en la enseñanza, etc.; sin embargo, encontramos pocas alusiones respecto al cómo enseñar. A continuación describiremos las diferentes opciones metodológicas a utilizar en la enseñanza del Tenis de Mesa, teniendo en cuenta los Métodos de enseñanza de los que se dispone hoy en día en las Ciencias de laActividad Física y el Deporte.

Tradicionalmente la enseñanza del Tenis de Mesa se ha basado en una Técnica de Enseñanza mediante la instrucción directa, en una Estrategia en la Práctica analítica y en Estilos de Enseñanza como el mando directo y la asignación de tareas; aspectos propios del Modelo tradicional (centrado en la técnica). Como alternativa, el Modelo global (centrado en la táctica) aboga por la utilización de una Técnica de Enseñanza basada en la indagación, por una Estrategia en la Práctica global y por Estilos de Enseñanza menos directivos, como por ejemplo, el descubrimiento guiado y la resolución de problemas.

En este sentido se han realizado investigaciones en los deportes de raqueta acerca de la idoneidad de unos métodos de enseñanza respecto a otros. McPherson y French (1991), estudiaron los cambios en las estrategias cognitivas como resultado de dos tipos de instrucción en tenis. En su primer experimento (instrucción directa) encuentran una mejora en la técnica y también en el juego. En el segundo experimento (instrucción estratégica) concluyen que no se produjeron mejoras en la ejecución técnica hasta que no se incluyó la enseñanza centrada en ésta. Por otro lado, French, Werner, Rink, Taylor, y Hussey (1996), estudiaron los efectos producidos a través de la enseñanza basada en la técnica, en la táctica y en una combinación de ambas en bádminton durante tres semanas en chicos y chicas de 14-15 años (edades correspondientes a la etapa de Secundaria). Estos autores afirman que se produjeron mejoras con las tres formas de instrucción en la técnica y en el juego. Se produjo 
una mejora de la técnica sin instrucción directa, lo cual difiere de lo encontrado por McPherson y French (1991). Sin embargo, la mejora en los conocimientos tácticos fue escasa en cualquiera de los grupos a los que se les aplicó las distintas intervenciones, quizás, según estos autores, debido al escaso tiempo de aplicación del tratamiento. Por este motivo, French, Werner, Taylor, Hussey, y Jones (1996), realizaron un estudio similar al anterior pero con una duración de seis semanas, aunque los resultados no difirieron significativamente de los encontrados en el trabajo anterior.

Respecto al Tenis de Mesa, Raab, Masters, y Maxwell (2005) efectúan un estudio con jugadores de este deporte cuya media de edad es de 11'4 años, edad que se corresponde con el final de la etapa de Primaria y principios de Secundaria. Estos autores concluyen que se producen mejoras en el cómo (técnica) y en las decisiones (táctica) como consecuencia de la aplicación de un entrenamiento combinado de técnica y táctica. También Sève, Saury, Ria, y Durand (2003) tras analizar la actividad de jugadores durante una interacción competitiva en Tenis de Mesa, manifiestan que ésta tiene unas características específicas referente a la toma de decisiones (táctica), que difícilmente pueden ser reproducidas en el entrenamiento. Proponen que la práctica y el entrenamiento han de ser diseñados para desarrollar habilidades de investigación y de resolución de problemas en los jugadores. Recientemente Tielemann (2007) realizó tres estudios (el segundo con adolescentes novatos con una media de edad de 13 ' 9 años) con jugadores aprendices, encontrando mejoras significativas mediante una instrucción centrada en la táctica (aprendizaje implícito) en el Tenis de Mesa con jugadores principiantes. Esta autora se basó en una instrucción de analogía en la que no se dan a los discentes instrucciones explícitas sobre lo que tienen que realizar, y donde la capacidad cognitiva y de resolución de problemas se ven altamente demandadas. Un ejemplo de analogía en el tenis de mesa sería decirle al niño o niña que tiene que golpear la bola igual que si estuviera lanzando un frisbee (en la enseñanza del top spin de revés). Otro estudio similar al anterior es el llevado a cabo por ChuMin y Masters (2001) en el que se muestran los beneficios de la enseñanza del Tenis de Mesa a través del aprendizaje por analogías como medio de aprendizaje implícito.

Nosotros adoptaremos en nuestra propuesta una posición ecléctica, en la que apostaremos por una u otra opción en función de aspectos como la edad de los jugadores/alumnos, tipo de tarea a enseñar, características de la estructura y lógica interna del Tenis de Mesa, etc.; sin embargo, somos conscientes de la dificultad que entraña la utilización del ModeloAlternativo (centrado en la táctica) en un deporte individual como el Tenis de Mesa; pero creemos, no obstante, y basándonos en las consideraciones realizadas por los autores anteriores en relación con los deportes de raqueta (tenis y bádminton) y, más concretamente, con el Tenis de Mesa, añadiendo también las de autores como Méndez (2004) y Turner y Martinek (1999) en otros deportes, que existen numerosas características de este Modelo que pueden ser empleadas en la enseñanza de nuestro deporte, lo cual intentaremos ilustrar con algunos ejemplos.

\section{Propuesta metodológica}

Nos referimos, pues, en este apartado, al ¿cómo enseñar? Es decir: a la forma de enseñar los medios técnico-tácticos del Tenis de Mesa, pero también, no lo olvidemos, a la manera de desarrollar cognitivamente a nuestros jugadores/alumnos. De los distintos Métodos relacionados con la enseñanza del Tenis de Mesa, nosotros nos vamos a centrar en los que hacen referencia a la Técnica de Enseñanza, a la Estrategia en la Práctica y a los Estilos de Enseñanza, aunque también haremos referencia a aspectos y consideraciones metodológicas a tener en cuenta en la enseñanza del Tenis de Mesa.

\subsection{Técnica de enseñanza}

Para desarrollar este apartado nos basaremos en Vannier y Fait, citados por Delgado (1993). En el Tenis de Mesa existen gestos técnicos o modelos de ejecución que han demostrado su eficacia; esto hace que se tomen como referencia para la enseñanza de los distintos medios. En este sentido, siempre vamos a intentar alcanzar ese ideal que propone el modelo; esto lo podemos hacer exponiendo éste al jugador/alumno que aprende (a través de la demostración propia o de otro jugador, ya sea en directo o mediante una grabación en video), lo cual es característico del Modelo Tradicional (Técnica de enseñanza por instrucción directa, por imitación o por modelos); no obstante, también es posible acercar al jugador o alumno a ese modelo progresivamente a través de la Técnica de Enseñanza de la indagación, en la que el chico o chica ha de descubrir los aspectos más relevantes del modelo, tal y como exponemos en los siguientes ejemplos:

- Si pretendemos enseñar el corte de derecha o de revés, tras haber enseñado el peloteo sin efecto, el chico o chica podrá ir descubriendo cómo orientar la pala para que el efecto cortado no haga que su golpe (en principio con la pala vertical respecto a la mesa) envíe la pelota contra la red. En este sentido, se le puede preguntar al chico o chica: ¿qué ocurre cuando inclinas un poco la pala hacia arriba (supinación del antebrazo)?, ¿la pelota, se va hacia arriba o hacia abajo?, etc. De esta forma, estamos fomentando el aprender a pensar sobre la causa y el efecto que tienen determinadas acciones y la trayectoria de la pelota cuando ésta lleva o no efecto, lo cual será de suma importancia, no solo para este aprendizaje en concreto, sino para adquisiciones ulteriores.

- Enseñanza del bloqueo activo. Normalmente, en las primeras experiencias de los chicos y chicas con el blocaje, la bola suele tomar una trayectoria excesivamente alta al volver al campo contrario, incluso a veces se marcha por la línea de fondo de éste sin botar en la mesa. Esto se produce por la tendencia natural del niño o niña a poner la pala en posición vertical respecto a la mesa. Una vez que el alumno o alumna haya tenido estas primeras experiencias, en vezdemostrarles directamente cómo ha de actuar, podemos hacerle preguntas acerca del comportamiento de la bola tras golpear su pala: ¿hacia dónde se ha ido la bola, hacia arriba o hacia abajo? ¿Por qué crees que ocurre eso? ¿Qué crees que pasaría si inclinases la pala hacia la mesa (pronación)?, etc. De esta forma llegamos al modelo pero a través del razonamiento cognitivo, lo cual hará que el aprendizaje sea más duradero y significativo.

Si somos capaces de combinar adecuadamente el uso de una y otra Técnica de Enseñanza conseguiremos que nuestros alumnos/jugadores, además de aprender el gesto técnico, comprendan el por qué de la utilización de ese gesto y no de otro, y de por qué se debe realizar de esa forma y no de otra. Esto, junto con el engarce del aprendizaje con los conocimientos o ideas anteriores, y según Gómezy Mauri (1991), es la clave de todo aprendizaje significativo. Este para qué sirve lo que se hace (intencionalidad) es la base del buen aprendizaje (Mahlo, 1974, citado por Martín (2005). Se fomentará así el feed-back interno o retroalimentación -aspecto importantísimo en la enseñanza deportiva según Castejón y Pérez-Llantada (1999)- y cada vez que cometan un error, podrán analizar con mayor eficacia la causa del mismo, ya que van adquiriendo conocimientos y experiencias que les ayudarán a analizar cada situación y a tomar decisiones al respecto. Es lo que Sampedro (1999) denomina inteligencia motriz. Les estamos enseñando a pensar por sí solos, a ser independientes y autodidactos, a la vez que se convierten en protagonistas de su propio aprendizaje, lo cual refuerza su motivación intrínseca, ya que sienten satisfacción al entender sus fallos y poder tomar las medidas oportunas para subsanarlos en las próximas ocasiones. Entenderán, desde el principio, que la trayectoria y el efecto de la bola, por ejemplo, son aspectos de gran relevancia a tener en cuenta para jugar correctamente al Tenis de Mesa. En este sentido se expresa Pérez(2005), cuando manifiesta que muchos jugadores cuando cometen un error achacan su fallo a una mala ejecución técnica, no dándose cuenta de que ésta solo es un medio por el que nuestra mente, tras haber analizado una bola determinada, actúa dando la orden de realizar el gesto. De esta manera, de nada sirve realizar un gesto técnico de forma perfecta, si no somos capaces de leer adecuadamente las connotaciones de la trayectoria y el efecto de la bola; esto nos llevará, irremediablemente, a errar.

Como podemos comprobar, la Técnica de enseñanza basada en la indagación o resolución de problemas es más educativa, ya que implica, 
Tabla 1. Ventajas e inconvenientes de las técnicas de enseñanza, según Sáenz-López (1997, p. 145)

\begin{tabular}{|c|l||l||}
\hline \hline \multirow{3}{*}{ Instrucción directa } & \multicolumn{1}{|c|}{ VENTAJAS } & \multicolumn{1}{|c|}{ INCONVENIENTES } \\
\cline { 2 - 3 } & - Más eficiente y rápida & - Más difícil individualizar \\
& - Más fácil la organización y el control & - Papel del alumno/a receptivo y pasivo \\
\hline \hline \multirow{3}{*}{ Indagación } & - Aprendizajes técnicos más perfectos & \\
& - Mayor implicación cognitiva & - Aprendizajes más lentos \\
& - Libertad, creatividad y espontaneidad & - Técnicamente menos perfectos \\
& - Mayor individualización & - Requiere mayor preparación del profesor/a \\
\hline
\end{tabular}

fundamentalmente, a los mecanismos de cognición y decisión, además del mecanismo de ejecución, lo cual concuerda con lo expuesto por autores como Le Boulch (1991) y Contreras (1996). Quizás, adolece de cierta dificultad a la hora de poner en práctica, y de que los aprendizajes son más lentos, pero la motivación, nivel de retención y de significación que produce, son suficientes razones para utilizarla. En el Tenis de Mesa el jugador o alumno tiene que estar resolviendo problemas constantemente; además, el nivel de incertidumbre es elevado, con lo que el aprendizaje de estrategias cognitivas de análisis, abstracción y generalización se torna imprescindible. En relación con lo anterior, Mosston (1988, p. 239), nos dice: $<<$ Cuanto más amplia sea la experiencia del individuo en la solución de problemas referentes a un área de actividad, tanto mayores serán las posibilidades de transferirlas a otras áreas. En este sentido, la transferencia se relaciona con las dimensiones emocional y cognitiva que con tanta frecuencia condicionan las respuestas físicas >>. Sáenz-López (1997, p. 145), expone resumidamente las ventajas e inconvenientes de las Técnicas de enseñanza comentadas (tabla 1).

\subsection{Estrategia en la práctica}

Para Delgado (1993), la Estrategia en la práctica es la manera de presentar una actividad o tarea el profesor/entrenador. Este autor nos habla de Estrategia en la práctica global, Estrategia en la práctica analítica y Estrategia en la práctica mixta.

Estrategia en la práctica analítica: Se trata de descomponer una tarea o un gesto técnico en partes y enseñarlas por separado. Sánchez(1992), distingue los siguientes tipos de Estrategia en la práctica analítica:

- Analítica pura: la tarea se divide en partes y se comienza por la que el profesor/entrenador considera más importante; después la siguiente parte y así sucesivamente hasta hacer, al final, la tarea globalmente. Partes: A, B, Cy D. Se realiza, por ejemplo, primero la parte C; después la $\mathrm{D}$; después la $\mathrm{A}$; y después la $\mathrm{B}$. Se termina realizándola de forma completa: $\mathrm{A}+\mathrm{B}+\mathrm{C}+\mathrm{D}$. Ejemplo: $1^{\circ}$ peloteo de derecha; $2^{\circ}$ peloteo de revés; $3^{\circ}$ top spin de derecha; $\mathrm{y} 4^{\circ}$ partido $1 \mathrm{x} 1$.

- Analítica secuencial: se dividen las partes y se comienza en el mismo orden que aparecen las partes en la actividad. $\mathrm{Al}$ final se realiza globalmente. Suele utilizarse en la enseñanza de los gestos técnicos de los deportes. Partes: A, B, C y D. Se realiza primero la parte A; después la B; después la ; después la $\mathrm{D}$; y al final se realizan todas conjuntamente. Ejemplo: $1^{\circ}$ agarre de la pala; $2^{\circ}$ posición de las piernas; $3^{\circ}$ orientación del cuerpo; $4^{\circ}$ posición inicial del brazo ejecutor; $5^{\circ}$ recorrido del brazo en el golpeo de derecha; $6^{\circ}$ posición final del brazo y golpeo de la bola; $7^{\circ}$ se realiza todo el movimiento completo.

- Analítica progresiva: se comienza por una parte y cuando ésta se domina se añade otra y cuando estas dos se realicen correctamente, se añade otra parte y así sucesivamente hasta que se ejecute la tarea completa. Al final se practica la tarea de forma global. Partes: A, B, Cy D. Suele utilizarse en la enseñanza de los gestos técnicos de los deportes. Ejemplo para jugador diestro: $1^{\circ}$ golpe de revés (en el lado de revés); $2^{\circ}$ golpe de derecho (en el lado de derecho); $3^{\circ}$ golpe de revés, desplazamiento lateral hacia el lado derecho, golpe de derecho y desplazamiento lateral hacia el lado izquierdo; $4^{\circ}$ así sucesivamente.

Estrategia en la práctica global: En esta ocasión la tarea se presenta en su totalidad y no se divide en partes. Sánchez (1992), nos habla de los siguientes tipos de Estrategia en la práctica global: global pura, global polarizando la atención y global modificando la situación real.

- Global pura: se trata de la realización total de la tarea. En el Tenis de mesa sería jugar un partido de $1 \times 1$ ó de $2 \times 2$. Se aconseja utilizarla al final de una progresión de enseñanza, es decir, al final de la sesión para practicar e interiorizar los aprendizajes anteriores. Por eso, al final del entrenamiento se suelen jugar algunos partidos y también se participa en competiciones preparatorias para otras oficiales o de mayor importancia.

- Global modificando la situación real: en esta ocasión se realiza la tarea modificando las condiciones reales de práctica. Esto se puede hacer, bien para facilitar la realización de la tarea, bien para dificultarla; según nos interese. Como ejemplos podemos citar jugar un 1x1 sin red, jugar un 2x1, jugar un partido solo cortando, o solo de pico a pico (cruzado o paralelo)... Normalmente estas variaciones se deben hacer en función del nivel de los jugadores, del objetivo de enseñanza que nos propongamos conseguir, etc.

- Global polarizando la atención: Se le pide al jugador o alumno que realice la tarea de forma global y que se fije en algún aspecto o parte concreta de la ejecución. Ejemplo: golpe de derecho, polarizando la atención en golpear la bola en el punto más alto de trayectoria de la misma.

Estrategia en la práctica mixta: se trata de combinar la Estrategia en la práctica global y la Estrategia en la práctica analítica. Como ejemplo podemos citar la realización de un 1x1 cortando en toda la mesa (global modificando la situación real), después realizar cortes cruzados de revés y después cortes cruzados de derecho (analítico) y después realizar un $1 \mathrm{x} 1$ puntuando doble el punto conseguido mediante el corte de revés (global polarizando la atención y modificando la situación real). La estrategia mixta puede resultar de especial interés en tareas complejas de baja organización, según Sáenz-López (1997), como el Tenis de Mesa.

Existe, pues, una gran variedad de estrategias metodológicas que pueden servirnos para la enseñanza del Tenis de Mesa. Estará en nuestras manos el elegir una u otra, según nos interese; no obstante, se recomienda no abusar de las Estrategias analíticas, ya que se dificulta la aplicación posterior al juego real al existir una descontextualización del aprendizaje. Por este motivo, la estrategia global ha de estar presente en todos los entrenamientos. Además, cuando la tarea se practica de forma global la motivación de los practicantes aumenta, ya que la exigencia en la resolución de problemas es mayor, así como también creceel grado de incertidumbre; y esto no es otra cosa que la esencia del Tenis de Mesa. A continuación, y basándonos en Sáenz-López (1997, p. 133), exponemos unas consideraciones quenos ayudarán en la utilización correcta de las distintas Estrategias en la práctica en el Tenis de Mesa (tabla 2).

\subsection{Estilos de enseñanza}

Estaríamos ante la forma peculiar que tiene cada profesor a la hora de organizar la clase, relacionarse con sus alumnos, tomar decisiones relativas a la organización y control de la clase, etc. En este campo, destacan los estudios realizados por Mosston y Ashworth (1993), los cuales van a distinguir entre estilos directivos y estilos de descubrimiento. No obstante, Delgado (1991) profundiza algo más y distingue los siguientes: estilos de enseñanza tradicionales; estilos de enseñanza que fomentan la individualización; estilos de enseñanza que posibilitan la 
Tabla 2. Análisis de los factores que influyen en la utilización de las estrategias en la práctica según Sáenz-López (1997, p. 133), aplicado al Tenis de Mesa

\begin{tabular}{|c||c||l||l||}
\hline \hline \multirow{2}{*}{ Complejidad tarea } & \multicolumn{1}{|c||}{ GLOBAL } & \multicolumn{1}{|c||}{ ANALÍTICA } & \multicolumn{1}{c||}{ MIXTA } \\
\cline { 2 - 4 } & $\begin{array}{l}\text { Tareas simples } \\
\text { Ejemplo: corte de revés }\end{array}$ & $\begin{array}{l}\text { Tareas complejas } \\
\text { Ejemplo: corte de revés, } \\
\text { giro y top spin de derecho }\end{array}$ & $\begin{array}{l}\text { Tareas simples de alta } \\
\text { organización } \\
\text { Ejemplo: golpe de derecho } \\
\text { (lado derecho) y golpe } \\
\text { revés (lado de revés) }\end{array}$ \\
\hline \hline Organización tarea & $\begin{array}{l}\text { Tareas de baja organización } \\
\text { Ejemplo: 1x1 cortando }\end{array}$ & $\begin{array}{l}\text { Tareas de alta organización } \\
\text { Ejemplo: corte de revés }\end{array}$ & $\begin{array}{l}\text { Tareas complejas y de } \\
\text { baja organización } \\
\text { Ejemplo: 2x2 }\end{array}$ \\
\hline \hline Edad & Todas las edades & $\begin{array}{l}\text { A partir de los 11-12 años. } \\
\text { Antes en forma de juego }\end{array}$ & $\begin{array}{l}\text { A partir de 10-11 años. } \\
\text { Antes en forma de juego }\end{array}$ \\
\hline \hline Objetivo & $\begin{array}{l}\text { Más educativo } \\
\text { Iniciación }\end{array}$ & $\begin{array}{l}\text { Más eficacia, rendimiento y } \\
\text { perfeccionamiento }\end{array}$ & $\begin{array}{l}\text { Rendimiento } \\
\text { perfeccionamiento y alto } \\
\text { rendimiento }\end{array}$ \\
\hline \hline Formación entrenador/a & Requiere mucho dominio & Requiere dominio básico & Requiere mucho dominio \\
\hline \hline
\end{tabular}

participación del alumno en la enseñanza; estilos de enseñanza que propician la sociabilidad; estilos de enseñanza que comportan la implicación cognoscitiva directa del alumno en su aprendizaje; y estilos de enseñanza que estimulan la creatividad.

Nosotros nos ocuparemos solo de aquellos estilos de enseñanza que tengan, a nuestro juicio, una mayor posibilidad de aplicación a la enseñaza del Tenis de Mesa. En este sentido, los dividiremos, en estrecha relación con las Técnicas de enseñanza descritas más arriba, en estilos de enseñanza tradicionales y en estilos de enseñanza que fomentan la indagación y la participación cognitiva del jugador o alumno.

\subsection{Estilos de enseñanza tradicionales}

Mando directo modificado: según Delgado (1991), este estilo es menos rígido que el mando directo tradicional. Se basa en que el profesor/ entrenador es el experto y el que toma todas las decisiones; se persigue un modelo y se adiestra al alumno/a para la consecución del mismo; no existe individualización, ya que el alumno no tiene elección de respuesta, porque éste le viene dada de antemano (modelo); la organización de la clase es formal y existe gran control por parte del profesor/entrenador; la secuencia de enseñanza es la siguiente: a) explicación-demostración; b) ejecución; yc) evaluación-corrección por parte del profesor/entrenador.

Ejemplo de aplicación: el profesor/entrenador explica y demuestra el golpe de revés, el jugador o alumno lo realiza y el profesor/entrenador corrige los errores cometidos, y así en cada una de las repeticiones ordenando, el comienzo y el final de la actividad.

Asignación de tareas: siguiendo a Mosston (1988), se enseña a través del modelo; el profesor/entrenador transfiere algunas decisiones al alumno, pero sigue decidiendo el antes (tarea) y el después (evaluación), aunque deja cierta libertad al alumno en lo referente al ritmo de ejecución, períodos de pausa, etc. Como vemos, este estilo permite mayor individualización.

Ejemplo de aplicación: el profesor explica el corte de derecho encima de la mesa, los jugadores/alumnos lo realizan en sus respectivas mesas varias veces seguidas, descansando cuando crean conveniente y el profesor/entrenador va pasando por las mesas y va corrigiendo los fallos.

Enseñanza recíproca: se basa en el hecho de que el entrenador/ profesor no siempre puede atender a un gran número de jugadores/ alumnos, con lo que se contempla la posibilidad de que los propios jugadores/alumnos se ayuden entre sí. Por tanto, podemos aprovechar los conocimientos y experiencias de nuestros alumnos más aventajados y asignarles un rol en la enseñanza de aquellos compañeros que lo demanden. Esto fomentará una gran motivación de todos lo implicados. Previamente, es aconsejable que el profesor/entrenador instruya a los alumnos que van a ejercer de profesor/entrenador. La organización de la clase será por parejas. El profesor decide el antes (tarea), pero deja a los alumnos mayor participación e individualización en su enseñanza en el durante y en el después.

Ejemplo de aplicación: se reúne a los jugadores más aventajados y se les comunica que la tarea a enseñar es la del top spin de derecha paralelo; se les dice que presten atención en la colocación previa de las piernas y en el roce de la pala con la bola; y que instruyan, sobre todo, a sus parejas en esos aspectos. Tras varios minutos de práctica, el profesor/entrenador se acerca a cada mesa y da orientaciones al que está corrigiendo a su compañero, nunca al ejecutante, para no quitarle protagonismo al jugador/entrenador.

\subsection{Estilos de enseñanza que fomentan la participación} cognitiva

Descubrimiento guiado: en realidad, se trata de un estilo intermedio entre las dos Técnicas de enseñanza, ya que reúne características de las dos: el profesor/entrenador sigue tomando las decisiones relativas a la programación y evaluación, pero las actividades se desarrollan a través de la indagación o descubrimiento. Se caracteriza porque no se basa en el modelo, pero en ocasiones lo busca; el jugador o alumno participa cognitivamente en su aprendizaje; y el profesor va guiando al jugador o alumno, haciéndole preguntas que fomenten el pensamiento reflexivo y de análisis, sugiriéndoles respuestas adicionales, etc., hasta que éste consiga solucionar el problema planteado en la tarea. Siguiendo a Derri y Pachta (2007), este estilo de enseñanza favorece el aprendizaje motor y la retención, lo cual avala su utilización.

Ejemplo de aplicación: desplazamientos en la mesa. Un jugador o alumno practica en la mesa un corte de revés, después un corte de derecho, después corte de revés, y así sucesivamente. El profesor para el juego y le pregunta: ¿Te sientes cómodo desplazándote de un lado para otro? ¿Crees que lo estás haciendo bien? ¿Te estás tropezando contigo mismo? ¿Cómo crees que podrías evitarlo?, etc. De esta forma, y poco a poco, llegaríamos a la conclusión de que para desplazamientos cortos y cerca de la mesa es aconsejable utilizar desplazamientos laterales hacia ambos lados. Esto tendrá mucha significación para el jugador o alumno, ya que aprenderá el por qué se debe realizar así, con lo que su motivación aumentará considerablemente y el aprendizaje será más duradero.

Resolución de problemas: este estilo de enseñanza es el más representativo de la enseñanza a través de la indagación o de la búsqueda. El jugador o alumno es el verdadero protagonista de su aprendizaje. En este caso, no se persigue un modelo. El profesor/entrenador propone un problema y el jugador o alumno tiene que encontrar la o las respuestas adecuadas. Se fomentan, así, una gran participación cognitiva y la autoevaluación. El profesor refuerza las respuestas. Aquí tenemos que decirque la investigación llevada a cabo por Bootsma, Houbiers, Whiting, y Wieringen (1991) en la enseñanza del tenis de mesa, sugiere que el 
entrenamiento bajo condiciones dinámicas favorece la experimentación y la búsqueda de estrategias óptimas durante la adquisición de una habilidad. Esta consideración refuerza el empleo de este estilo de enseñanza en la iniciación al tenis de mesa.

Ejemplo de aplicación: se le dice a un jugadoro alumno: tu adversario te saca desde su pico de revés hacia tu revés mediante un servicio largo y con efecto lateral, soluciona el problema: una vez practicado este ejercicio varias veces se le puede preguntar: ¿qué posibilidades de resto tienes? ¿Cuál crees que es la mejor opción de resto? ¿Por qué? De esta forma, se fomenta la inteligencia motriz y la anticipación; es decir: la táctica.

Libre exploración: en este caso el jugador o alumno es quien toma, prácticamente, todas las decisiones relativas a las actividades, ritmo de ejecución, espacios a utilizar, etc. Se fomenta así la creatividad y la individualización del aprendizaje. El profesor/entrenador establece unas normas mínimas de control, motiva la participación, refuerza la creatividad de sus alumnos y anota las respuestas más interesantes que dan sus jugadores/alumnos para más tarde orientar las actividades y tareas hacia la consecución de determinados objetivos o contenidos.

Ejemplo de aplicación a la enseñanza del Tenis de Mesa: este estilo de enseñanza puede ser utilizado en la fase de familiarización con los materiales del Tenis de Mesa (bola, pala y mesa), donde el niño juega libremente y el profesor se limita al control de contingencias y a facilitar el juego del niño, tomando nota de las actividades interesantes. Por ejemplo: forma de coger la pala de algún niño, golpeos continuos contra la pared o contra el suelo, etc. Después los jugadores/alumnos realizarán estas actividades.

\section{Consideraciones finales}

Para finalizar, exponemos a continuación un resumen de las consideraciones a tener en cuenta en la enseñanza del Tenis de Mesa:

- Existe la necesidad de utilizar una metodología de enseñanza del Tenis de Mesa que combine la instrucción centrada en la técnica y aquélla que se centra en la táctica. Teniendo en cuenta los estudios de Raab, Masters, y Maxwell (2005) y de Tielemann (2007), esta forma de enseñar el Tenis de Mesa sería conveniente para alumnos y alumnas de edades correspondientes a educación Primaria y de Secundaria

- Dada la importancia del entrenador/profesor en la iniciación deportiva, tal como afirman Tabernero, Márquez, y Llanos (2002), la formación de éstos respecto al modelo alternativo se torna fundamental. Esto mismo también es sugerido por Unierzyski y Crespo (2007), en relación con otro deporte de raqueta como el tenis.

- En lo que respecta a la Educación Primaria, tenemos que decir que el niño, en las edades de 8-10 años presenta una capacidad de aprendizaje global (Le Boulch, 1976, citado por Giménez y Rodríguez, 2006). Por tanto, en la iniciación al Tenis de Mesa en esta etapa ha de predominar laEstrategia en la práctica global, aspecto destacado por Torres y Carrasco (1999) en la enseñanza de otro deporte de raqueta como el tenis.

- En la enseñanza del Tenis de Mesa en la educación Primaria y Secundaria, es aconsejable una mayor utilización de la Técnica de enseñanza a través de la búsqueda y de los estilos de enseñanza que fomentan la indagación (Le Boulch, 1991; Contreras, 1996; Derri y Pachta, 2007); así favoreceremos el desarrollo de la capacidad cognitiva, los aprendizajes serán más significativos (se irán construyendo de forma engarzada y tendrán una aplicación o funcionalidad directa) y conseguiremos una mayor motivación de nuestros jugadores/alumnos, lo cual incrementará el aprendizaje (Chiu-Ju, Chien-Chih, Hsiu-Li, MeiChi, y Kuo-Chuan, 2005).

- Destacar también, la importancia que tiene una adecuada comunicación en la enseñanza del Tenis de Mesa. Es beneficioso para el aprendizaje en Tenis de Mesa, siguiendo a Todorov, Shadmehr, y Bizzi (1997), retroalimentar (feed-back) a nuestros jugadores, lo cual ha de hacerse de forma clara, concisa, positiva y objetiva. También parece conveniente fomentar el conocimiento de resultados interrogativo, dejando a un lado los gritos, los castigos y otras formas poco educativas, (Castejón y Pérez-Llantada, 1999).
- No se debe caer en el error de enseñar una técnica muy rígida y automatizada, puesto que, al ser el Tenis e Mesa un deporte de situación (acíclico), se perdería capacidad para resolver los problemas planteados en un partido (Minc, 2001).

- Las actividades y ejercicios que planteemos deben, en su mayoría, aproximarse progresivamente a las condiciones reales de juego (Angelescu, 1988; Sève, Saury, Theureau, y Durand, 2002; Sève, Saury, Ria y Durand, 2003; Poolton, Masters, y Maxwell, 2006). Se trata de la enseñanza contextual del Tenis de Mesa. De esta manera, el jugador o alumno comprenderá el porqué de cada actividad, gesto, secuencia de movimientos, aspectos de tener en cuenta antes de golpear una bola, etc.; es decir: comprenderá el Tenis de Mesa.

- El juego es fundamental para el desarrollo y el aprendizaje del niño. Se debe utilizar como estrategia metodológica y también como aprendizaje en sí mismo, ya que el Tenis de Mesa es, en esencia, juego.

- La capacidad de concentración de los chicos y chicas se presenta mermada si la comparamos con la de un adulto, por lo que las actividades y juegos han de ser variados y de corta duración.

- El niño presenta un gran egocentrismo, con lo que necesita estar en contacto con la pala, con la pelota, pero también con la mesa, ya que su principal objetivo es satisfacer su necesidad de jugar al Tenis de Mesa $<<$ en la mesa $>>$.

- Siguiendo a Sáenz-López (1997), los dos pilares básicos del aprendizaje motor son: la motivación y la práctica; se ha de fomentar, pues, la motivación de los niños y niñas que están aprendiendo, no solo la motivación externa, sino también la que proviene del interior de la persona que aprende; es decir: la motivación intrínseca. Por otro lado, es conveniente tener en cuenta que solo se aprende practicando; jugando al Tenis de Mesa (esto parece evidente, pero, a veces, a los entrenadores y profesores se nos olvida).

Quisiéramos terminar nuestro trabajo expresando que para mejorar la enseñanza de los deportes de raqueta en general, y del Tenis de Mesa en particular, hacen falta nuevas y abundantes investigaciones y estudios relacionados con éstos, sobre todo, en relación con los modelos, técnicas, estrategias y estilos de enseñanza aplicados a la enseñanza de estos deportes, tanto en el ámbito educativo como en el deportivo.

\section{Bibliografía}

Angelescu, N. (1988). El Tenis de Mesa. Barcelona: Juventud, S. A. Bermejo, J. L. (1991). Tenis de Mesa. Madrid: F.E.T.M. C.O.E.

Bootsma, R. J., Houbiers, M. H. J., Whiting, H. T.A. y Wieringen, P. C. W. (1991). Acquiring an attacking forehand drive: the effects of static and dynamic environmental conditions. Research Quarterly for Exercise and Sport, 62, 3, 276-284.

Castejón, F. J. y Pérez-Llantada, M. C. (1999). La utilización del conocimiento de los resultados interrogativo en una habilidad deportiva. Habilidad Motriz, 13, 5-8.

Contreras, O. R. (1996). El deporte educativo(II). La iniciación deportiva en el diseño curricular base de educación primaria. Revista de Educación Física. Renovación de teoría y práctica, 62, 33-37.

Chiu-Ju, L., Chien-Chih, C., Hsiu-Li, T., Mei-Chi, C. y Kuo-Chuan, T. (2005). The relationships between learning motivation and learning outcomes of table tennis in college physical education. Research Quarterly for Exercise and Sport, 76 (Suppl.), 1, A82.

Chu-Min, L. y Masters, R. S. W. (2001). Analogy learning: a means to implicit motor learning. Journal of Sports Sciences, 19, 5, 307-319.

Delgado, M.A.(1991).Haciauna clarificación conceptual de los términos de la educación física y el deporte. Revista de Educación Física. Renovación de teoría y práctica, 40, 2-10.

Delgado, M. A. (1993). Los métodos didácticos en Educación Física. En AA.VV. (Ed.), Fundamentos de Educación Física para Enseñanza Primaria, Vol. II (pp. 1045-1066). Barcelona: INDE.

Derri, V. y Pachta, M. (2007). Motor skills and concepts acquisition and retention: a comparison between two styles of teaching. International Journal of Sport Science, 3, 9, 37-47. 
French, K. E., Werner, P. H., Rink, J. E., Taylor, K. y Hussey, K. (1996). The effects of a 3-week unit of tactical, skill, or combined tactical on skill instruction on badminton performance of ninthgrade students. Journal of Teaching in Physical Education, 15, 4, 418-438.

French, K. E., Werner, P. H., Taylor, K., Hussey, K. y Jones, J. (1996). The effects of a 6-week unit of tactical, skill, or combined tactical and skill instruction on badminton performance of ninth-grade students. Journal of Teaching in Physical Education, 154, $439-463$

Giménez, F. J. y Rodríguez, J.M. (2006). Buscando el deporte educativo. ¿Cómo formar a los maestros? Retos. Nuevas tendencias en Educación Física, Deporte y Recreación, 9, 40-45.

Gómez, I. y Mauri, T. (1991). La funcionalidad del aprendizaje en el aula y su evaluación. Cuadernos de Pedagogía, 188, 28-32.

González, M. D. y Aznar, R. (2007). La importancia de las teorías implícitas del alumnado en la didáctica de los deportes de raqueta [Versión electrónica]. Revista Digital. Lecturas de E.F.y deportes, 107. Consultada el 04/05/07 en http://www.efdeportes.com/ efd107teorias-implicitas-del-alumnado-en-la-didáctica-de...

Le Boulch, J.(1991). El deporte educativo. Psicocinética y aprendizaje motor. Barcelona: Paidós.

Martín, L. J. (2005). Aprendizaje significativo. Tenis de Mesa. Revista oficial de la Federación Española de Tenis de Mesa, 100, 6.

McPherson, S. L. y French, K. E. (1991). Changes in cognitive strategies and motor skill in tennis. Journal of Sport and Excercise Psychology, $13,26-41$.

Méndez,A. (2004). Técnicas de enseñanza en la iniciación al baloncesto. Barcelona: INDE.

Minc, F. (2001). Tenis de mesa: fundamentos metodológicos para profesores [Versión electrónica]. Lecturas: Educación física y Deportes, Revista digital, 39. Consultada el 21/08/02 en www.efdeportes.com/efd39/tmesa1.htm.

Mosston, M. (1988). La enseñanza de la educación física. Barcelona: Paidós.

Mosston, M. y Ashworth, S. (1993). La enseñanza de la educación física. La reforma de los estilos de enseñanza. Barcelona: Hispano Europea.

Pérez, V. (2005). Del Big-Bang al ping-pong. Sevilla: Wanceulen.

Poolton, J. M., Masters, R. S. W. y Maxwell, J. P. (2006). The influence of analogy learning on decision-making in table tennis: evidence from behavioural data. Psychology of Sport and Exercise 7677-688

Raab, M., Masters, R. S. W. y Maxwell, J. P. (2005). Improving the $<<$ how $>>$ and $<<$ what $>>$ decisions of elite table tennis players. Human Movement Science, 24, 326-344.
Robles, J. (2005). El deporte en las clases de educación física en la E.S.O. de la provincia de Huelva [Versión electrónica]. Wanceulen E. F. Digital, 1. Consultada el 06/05/07 en http:// www.wanceulen.com/revista/nos.anteriores/numerol.diciembre05/ articulos/articulo\%201-7.htm.

Sáenz-López, P. (1997). La educación física y su didáctica. Manual para el Profesor. Sevilla: Wanceulen.

Salina, F. y Viciana, J.(2006). La planificación de los bloques de contenidos de laEducaciónFísica en Educación Secundaria Obligatoria[Versión electrónica]. Cienciay Deporte, 3. Consultada el 21/04/07 en http:/ /cienciaydeporte.net/articulo 0306 1.php.

Sampedro, J. (1999). Fundamentos de táctica deportiva. Análisis de la estrategia de los deportes. Madrid: Gymnos.

Sánchez, F. (1992). Bases para una didáctica de la educación física yel deporte. Madrid: Gymnos.

Sève, C., Saury, J., Ria, L. y Durand, M. (2003). Structure of expert player's activity during competitive interaction in table tennis. Research Quarterly for Exercise and Sport, 74, 1, 71-83.

Sève, C., Saury, J., Theureau, J. y Durand, M. (2002). Activity organization and knowledge construction during competitive interaction in table tennis. Cognitive Systems Research, 3, 501522.

Tabernero, B., Márquez, S. y Llanos, C. (2002). Elementos a analizaren el proceso de iniciación deportiva. Retos. Nuevas tendencias en Educación Física, Deporte y Recreación, 3, 9-15.

Tielemann, N. (2007). How instruction make SMART?, $12^{\text {th }}$ European Congress of Sport Psychology. Halkidiki, Greece: University of Thessaly. Greek Society of Sport Psychology.

Todorov, E., Shadmehr, R. y Bizzi, E. (1997). Augmented Feedback presented in a virtual environment accelerates learning of a difficult motor task. Journal of Motor Behavior, 29, 2, 147-158.

Torres, G y Carrasco, L. (1999). Estrategia en la práctica global contra analítica en deportes técnicos. Una propuesta de trabajo con tenis. En P. Sáenz-López, J. Tierra y M. Díaz (Eds.), XVII Congreso Nacional de Educación Física, Vol. II (pp. 907-911). Huelva: Universidad de Huelva. IAD.

Turner, A. P. y Martinek, T. J. (1999). An investigation into teaching games for understanding: effects on skill, knowledge, an games play. Research Quarterly for Exercise and Sport, 70, 3, 286-296.

Unierzyski, P.y Crespo, M. (2007). Review of modern teaching methods for tennis. International Journal of Sport Science, III, 7, 1-10. 